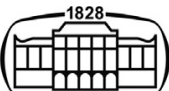

AKADÉMIAI KIADÓ

Journal of Behavioral Addictions

$10(2021) 2,302-313$

DOI:

$10.1556 / 2006.2021 .00011$

(c) 2021 The Author(s)

\section{FULL-LENGTH REPORT}

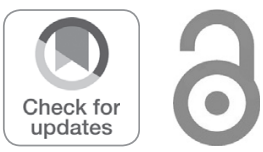

\title{
Social media 'addiction': The absence of an attentional bias to social media stimuli
}

\author{
KATIE THOMSON ${ }^{1}$, SIMON C. HUNTER ${ }^{2,3}$, \\ STEPHEN H. BUTLER ${ }^{1}$ and DAVID J. ROBERTSON ${ }^{1 *}$ (D) \\ ${ }^{1}$ School of Psychological Sciences and Health, University of Strathclyde, Glasgow, UK \\ ${ }^{2}$ Department of Psychology, Glasgow Caledonian University, Glasgow, UK \\ ${ }^{3}$ Graduate School of Education, University of Western Australia, Perth, Australia
}

Received: June 16, 2020 • Revised manuscript received: January 23, 2021 - Accepted: February 07, 2021

Published online: April 13, 2021

\begin{abstract}
Background and aims: Social media use has become a ubiquitous part of society, with 3.8 billion users worldwide. While research has shown that there are positive aspects to social media engagement (e.g. feelings of social connectedness and wellbeing), much of the focus has been on the negative mental health outcomes which are associated with excessive use (e.g. higher levels of depression/anxiety). While the evidence to support such negative associations is mixed, there is a growing debate within the literature as to whether excessive levels of social media use should become a clinically defined addictive behaviour. Methods: Here we assess whether one hallmark of addiction, the priority processing of addiction related stimuli known as an 'attentional bias', is evident in a group of social media users $(N=$ 100). Using mock iPhone displays, we test whether social media stimuli preferentially capture users' attention and whether the level of bias can be predicted by platform use (self-report, objective smartphone usage data), and whether it is associated with scores on established measures of social media engagement (SMES) and social media 'addiction' severity scales (BSNAS, SMAQ). Results: Our findings do not provide support for a social media specific attentional bias. While there was a large range of individual differences in our measures of use, engagement, and 'addictive' severity, these were not predictive of, or associated with, individual differences in the magnitude of attentional capture by social media stimuli. Conclusions: More research is required before social media use can be definitively placed within an addiction framework.
\end{abstract}

\section{KEYWORDS}

addictive behaviour, social media use, attention, attentional bias, individual differences

\section{INTRODUCTION}

There are currently 3.8 billion active social media users (Statista, 2020). With increased internet connectivity occurring across the world, individuals now have access to a suite of social media platforms (e.g. Facebook, Twitter, Instagram, Snapchat) which provide news, information, entertainment, an opportunity to post photos, express opinion, seek companionship, and maintain networks of friends and family (Ryan, Chester, Reece, \& Xenos, 2014; Whiting \& Williams, 2013). While engagement with social media can enhance feelings of social connectedness and wellbeing (Allen, Ryan, Gray, McInerney, \& Waters, 2014; Leist, 2013; McDaniel, Coyne, \& Holmes, 2012), research has largely focused on investigating the potential for negative mental health outcomes to arise from excessive use (Kircaburun et al., 2018; Shensa et al., 2017; Woods \& Scott, 2016). In such papers, and more generally, excessive social media use is increasingly being characterised as an addictive behaviour (Griffiths, 2000; Griffiths \& Kuss, 2017; Kuss \& Griffiths, 2017; LaRose et al., 2010; Ryan et al., 2014). However, it has not yet been recognised as such by the DSM-5 (APA, 2013) or ICD-11 (WHO, 2018) classification systems. 
The contemporary conceptualisation of addictive behaviour no longer focuses on the traditional substancerelated dependencies of alcohol (Enoch \& Goldman, 2002), smoking (Brown, Beard, Kotz, Michie, \& West, 2014), and the consumption of illegal drugs (Sofuoglu \& Kosten, 2006). Problematic behaviours related to gambling (Gainsbury, 2015), gaming (Kuss \& Griffiths, 2012), and internet use (Kuss \& Lopez-Fernandez, 2016) are regarded as displaying the same common set of underlying biopsychosocial addictive mechanisms (i.e. salience, mood modification, tolerance, withdrawal, conflict and relapse) as their chemical counterparts (Griffiths, 2005; 2018). With the growth of social media, it is now being argued that excessive use generates behaviours which meet the criteria for addiction (Griffiths \& Kuss, 2017; Ryan et al., 2014), with a potential prevalence rate of $4.5 \%$ being reported among a large sample of adolescents (Banyai et al., 2017).

The push towards characterising excessive social media use as a potentially addictive behaviour, which might produce negative mental health outcomes, has received mixed support in the literature. While there is some evidence that excessive use may be associated with lower self-esteem (Andreassen, Pallesen, \& Griffiths, 2017), and increased levels of depression (Lin et al., 2016; Shensa et al., 2017) and anxiety (Keles, McCrae, \& Grealish, 2020), other studies, some using longitudinal methods, have failed to replicate these effects (e.g. Coyne, Rogers, Zurcher, Stockdale, \& Booth, 2020; Heffer, Good, Daly, MacDonell, \& Willoughby, 2019; Houghton et al., 2018). What is clear, is that in contrast to substance-related addiction research, much less focus has been attributed to investigating whether excessive social media use can be detected through changes in cognitive processes such as selective attention. The present study does so by examining whether an 'attentional bias' (Anderson, 2013) to social media related stimuli is present in a group of social media users.

It is well-established that addictive behaviours are characterised by the priority processing of addiction related stimuli and that this is linked to the 'salience' mechanism noted above (Field \& Cox, 2008; Robbins \& Ehrman, 2004). In this way, users' top down goals bias their attention towards reward-based stimuli, and research suggests that this is underpinned by larger dopaminergic responses to addiction related stimuli in brain areas which mediate goaldirected behaviour (Franken, 2003; Haber, 2016), a key concept in the incentive-sensitization theory of addiction (Robinson \& Berridge, 1993, 2008). Moreover, once users' attention is inordinately captured by addiction related content, they appear to show a significantly higher degree of 'cue reactivity' (see Carter \& Tiffany, 1999), leading to a greater craving for, and propensity to engage in, the addictive behaviour in comparison to non-user neuro-typical controls (see Brand et al., 2019 and the I-PACE model of addictive behaviours). The combination of attentional bias and heightened cue reactivity is thought to play a major role in the development, maintenance, and relapse into addiction (Drummond, 2000; Papachristou, Nederkoorn, Giesen, \& Jansen, 2014).
Here we investigate the attentional bias component of the addictive process, and such biases have been reported across a range of substance-related addictions (see Field \& Cox, 2008 for a review), in addition, research has also shown that the magnitude of the effect displayed by a user could be indicative of the severity of their addiction. This individual differences approach has shown that attentional capture by alcohol-related images (e.g. a bottle of beer) is significantly greater in problem drinkers, as measured by self-report questionnaires of consumption and/or craving, compared to social and non-problem drinkers (Jones, Bruce, Livingstone, \& Reed, 2006; Sharma, Albery, \& Cook, 2001). This effect has been replicated in smokers (Waters et al., 2003), gamblers (Boyer \& Dickerson, 2003), and gamers (van Holst et al., 2012). That is, the greater the level of attentional bias (i.e. level of related dopaminergic change; Franken, 2003) that a user displays towards addiction related stimuli, the greater their self-reported rates of engagement with that substance.

Importantly, several recent papers have investigated these effects in non-substance related addictions such as gambling and problematic levels of internet use. Ciccarelli, Nigro, Griffiths, Cosenza, and D'Olimpio (2016) reported the presence of an attentional bias to gambling related images in problem gamblers using a modified version of the Posner Task. The magnitude of the bias was positively associated with scores on a gambling craving scale. Similarly, Jeromin, Nyenhuis, and Barke (2016) reported that a group of excessive gamers showed an attentional bias to computer related words using a modified Stroop task, in comparison to a group of non-gamers, a full individual differences analysis, in terms of addiction severity, was not reported. More recently, Pekal, Laier, Snagowski, Stark, and Brand (2018) reported the presence of an attentional bias to pornographic images in individuals with internet-pornography use disorder (IPD) using a visual probe task, with a significant positive relationship between symptom severity and the magnitude of the bias.

These studies of non-substance related addictions show that attentional biases appear to be present in such users (Ciccarelli et al., 2016; Jeromin et al., 2016; Pekal et al., 2018), and that individual differences in the magnitude of the attentional bias effect appear to indicate the level of addiction severity (Ciccarelli et al., 2016; Pekal et al., 2018). This suggests that as addiction progresses in these disorders, the 'incentive salience' of stimuli (Pekal et al., 2018) leads to an increase in the dopaminergic response for goal directed behaviour (Franken, 2003), which manifests itself cognitively as the increased attentional capture by addiction related content. While such effects appear to be present in nonsubstance related addictions such as gambling, gaming, and internet-pornography use, it is not yet clear whether such effects generalise to excessive social media use.

To our knowledge, only one paper has been published to date which has investigated the presence of an attentional bias in social media users. Nikolaidou, Fraser, and Hinvest (2019) reported an attentional bias, in the form of increased attentional dwell times, to social media related stimuli in a group of social media users. However, this finding could be 
limited by the task, which used over-sized social media images (e.g. twitter logo) paired with a control image on each trial. This type of display does not closely mirror real world user activity (i.e. searching a cluttered smartphone screen for a social media app). In addition, while the study did report a correlation between the level of urge to be online and dwell time to social media stimuli $(N=16 ; r=$ 0.703 ), the analysis was underpowered with only 16 participants in the sample. Therefore, further research is required to assess whether such a bias is present in social media users.

To that end, in the present study, we assess whether social media users show an attentional bias towards social media related stimuli, and by proxy addiction related changes to the brain's neurochemistry, and whether levels of social media use, engagement, and 'addictive' severity are associated with the magnitude of the effect. In a novel task, with high ecological validity, participants were required to view mock iPhone displays and to respond to the presence of a target app (Siri/Camera) while ignoring other distractor apps present in the display, an example is provided in Fig. 1. In condition 1, we assessed the extent to which the presence of a social media app, presented as one of the non-target distractors, captured users' attention and slowed target response times (i.e. a response time measure of attentional bias). In condition 2, we again presented social media apps as distractors, but this time on half of the trials these apps were also paired with a red ' 1 ' notification symbol, an example is also provided in Fig. 1. Notification symbols should also be highly salient images to social media users as they indicate that they have received feedback, or a 'reward' in addiction terminology, in the form of 'likes' or 'comments' to content that they have posted (see Scissors, Burke,
\& Wengrovitz, 2016). In addition, we also test whether the strength of any attentional bias can be predicted by levels of social media engagement through our own self-report questionnaire and objective smartphone data, and whether it is associated with scores on established measures of social media engagement (SMES) and social media 'addiction' severity (BSNAS, SMAQ).

\section{METHOD}

\section{Participants}

An a priori power analysis was conducted using $\mathrm{G}^{*}$ Power, it revealed that a minimum sample of 81 participants was required for our study in order to detect small effect sizes (alpha at 0.05 , power at 0.80 ). We recruited one hundred participants with a mean age of 20 years $(S D=2$, Range $=$ 17-32, 82 Female) from our psychology research participation pool. One participant was removed from the dataset as they reported not having a social media account with any of the four platforms of interest. This sample size compares favourably to previous studies of addiction/social media related attentional biases reported in the introduction. All participants reported having normal or corrected-to-normal vision.

For social media accounts, $96 \%$ of the sample were Facebook users, the rate was $71 \%$ for Twitter, $95 \%$ for Instagram, and $94 \%$ for Snapchat. Table 1 provides data from our questionnaire measures, these show a large degree of variability, including those at the top end of the response scales, in platform use, engagement, and 'addictive' severity.
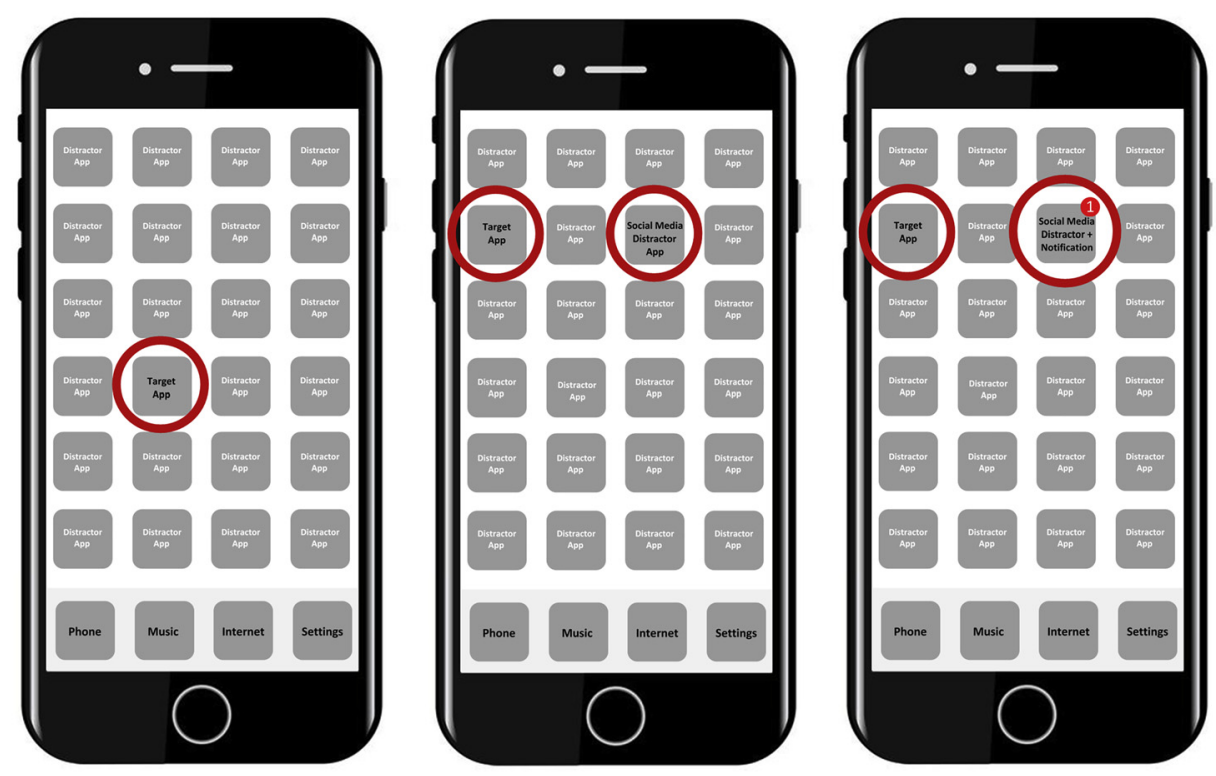

Fig. 1. Due to copyright reasons we are not able to show examples of the actual trials used in the experiment, these used real smartphone app icons and mirrored a regular iPhone display very closely. Here we present a close approximation of the layout of the display on trials in each condition. (Left) Example trial from the baseline condition with no social media distractor. (Middle) Example trial from the 'mere presence' condition with a social media distractor present. (Right) Example trial from the '+ notification' condition in which social media distractors, and non-social media control distractors, were overlaid with a notification symbol 
Table 1. Participant responses to measures of social media use, engagement, and 'addictive' severity

\begin{tabular}{|c|c|c|c|}
\hline & Mean & SD & Range \\
\hline \multicolumn{4}{|l|}{ Facebook users } \\
\hline Friends & 701 & 485 & $50-2,795$ \\
\hline Posts per week & 1 & 1 & $0-7$ \\
\hline Daily checks & 9 & 10 & $0-50$ \\
\hline Usage per week (min) & 194 & 186 & $0-771$ \\
\hline \multicolumn{4}{|l|}{ Twitter users } \\
\hline Friends (Followers) & 490 & 1,585 & $8-13,300$ \\
\hline Posts per week & 3 & 12 & $0-100$ \\
\hline Daily checks & 9 & 8 & $0-30$ \\
\hline Usage per week (min) & 176 & 175 & $0-840$ \\
\hline \multicolumn{4}{|l|}{ Instagram users } \\
\hline Friends (Followers) & 951 & 819 & $50-4,369$ \\
\hline Posts per week & 1 & 1 & $0-7$ \\
\hline Daily checks & 14 & 12 & $1-60$ \\
\hline Usage per week (min) & 284 & 225 & $33-1,320$ \\
\hline \multicolumn{4}{|l|}{ Snapchat users } \\
\hline Friends & 120 & 91 & $20-400$ \\
\hline Posts per week & 12 & 36 & $0-300$ \\
\hline Daily checks & 23 & 27 & $0-200$ \\
\hline Usage per week (min) & 365 & 356 & $6-1,500$ \\
\hline \multicolumn{4}{|c|}{ Bergen Social Networking Addiction Scale (BSNAS) } \\
\hline Self-report ratings & 19 & 5 & $6-29$ \\
\hline \multicolumn{4}{|c|}{ Social Media Addiction Questionnaire (SMAQ) } \\
\hline Self-report ratings & 35 & 9 & $8-51$ \\
\hline \multicolumn{4}{|c|}{ Social Media Engagement Scale (SMES) } \\
\hline Self-report ratings & 30 & 7 & $5-40$ \\
\hline
\end{tabular}

\section{Apparatus and materials}

Attentional capture task. The attentional capture task was programmed and run using E-Prime 2.0. Stimuli were presented and responses were recorded using a Dell PC with an 18inch screen. As seen in Fig. 1, each trial consisted of an image of an iPhone display populated with 28 apps. Care was taken to emulate a typical iPhone screen and so the bottom row of apps always consisted of iOS integrated apps (Phone, Music, Safari, Settings) presented in the same location across trials. The targets were the Siri app and the Camera app and were selected for their similarity in appearance and familiarity to users. There were 34 nontarget/non-social media 'filler' apps which were selected from the remaining integrated apps and a selection from the top 25 current most downloaded apps. The social media distractor apps consisted of Facebook, Twitter, Instagram and Snapchat. The iPhone was presented onscreen in its real-world size $(12.4 \mathrm{~cm} \times 5.9 \mathrm{~cm})$ and each app was 1 $\mathrm{cm} \times 1 \mathrm{~cm}$ in size. All stimuli were presented on a white background.

Social media questionnaire. This questionnaire was developed for this study. The first section asked participants to record which of the four social media accounts they had, and then for each of their accounts (as appropriate): how many friends/followers do you have, how many accounts do you follow, how many times a week do you post, and how many times a day do you check this platform. For the final question, participants were asked to report their social media app usage, in minutes, for the previous week, using the objective data (see Ellis, Davidson, Shaw, \& Geyer, 2019; Kaye, Orben, Ellis, Hunter, \& Houghton, 2020) collected by their smartphone integrated screen time use software $(75 \%$ were able to do so).

Bergen Social Networking Addiction Scale (BSNAS). The Bergen Social Media Addiction Scale (BSNAS) (Andreassen et al., 2016) is derived from the Bergen Facebook Addiction Scale (Andreassen, Torsheim, Brunborg, \& Pallesen, 2012). This scale utilises the six items of the addiction components model (Griffiths, 2005), salience, mood modification, tolerance, withdrawal, conflict and relapse, and assesses the use of social media over the past year. Questions included 'How often in the last year have you felt an urge to use social media more and more'. Participants were asked to rate all items on a 5-point Likert scale, ranging from 1 (very rarely) to 5 (very often). The internal consistency of the BSNAS was satisfactory with Cronbach's Alpha $=0.75$. For this questionnaire, and for those noted below a participant's score consisted of the sum of their Likert scale responses.

Social Media Addiction Questionnaire (SMAQ). This 8item questionnaire (Hawi \& Samaha, 2017) is derived from the Facebook Intrusion Questionnaire and assesses social media addiction and use. Questions included 'The thought of not being able to access social media makes me feel distressed'. Participants were asked to rate all items on a 7point Likert scale, ranging from 1 (strongly disagree) to 7 (strongly agree). The internal consistency was acceptable with Cronbach's Alpha $=0.82$.

Social Media Engagement Scale (SMES). The Social Media Engagement Scale (Przybylski, Murayama, DeHaan, \& Gladwell, 2013) is a 5-item scale which measures the extent to which people engage with social media in their daily lives. Questions included 'How often did you use social media in the last 15 minutes before you go to sleep?' Participants were asked to rate all items on an 8-point Likert scale ranging from 0 (not one day) to 7 (everyday). The internal consistency was satisfactory with Cronbach's Alpha $=0.76$.

\section{Procedure}

Each trial began with the presentation of a central fixation point for $500 \mathrm{~ms}$, followed by a mock iPhone display which remained onscreen until response (up to a maximum of $5,000 \mathrm{~ms}$ ), with a $1,500 \mathrm{~ms}$ blank interval following the search display. A target appeared on each trial and could be the Siri app or Camera app, and participants were instructed to try and detect the target as quickly and as accurately as they could while ignoring any other task irrelevant distracting information present in the display. Responses were made using keyboard keys 1 (Siri) and 2 (Camera). There were two practice blocks, each containing 12 trials. In the first practice block, the display showed only the target app in the absence of any other apps, while these were introduced 
in the second practice block to mirror the experimental displays. No social media distractor apps or notifications were displayed during practice.

Following practice, participants completed four experimental blocks, each containing 96 trials. Experimental blocks 1 and 2 (condition 1), presented social media distractor apps on $50 \%$ of trials, with the each of the four social media apps (Facebook, Twitter, Instagram, Snapchat) appearing on 12 occasions per block. The remaining $50 \%$ of trials contained no social media distractor app, and as such provided our baseline response times. The presentation of trials within blocks were randomised, and target/distractor type, target/distractor position within the array were counterbalanced within participants. The remaining 'filler' apps used to complete the 24 item display on each trial were randomly selected from a 34 item set, with each app only ever appearing once in a trial display.

Experimental blocks 3 and 4 (condition 2) followed the same procedure with the exception that here we overlaid a notification symbol over the social media distractor app on half of the trials (i.e. 24 trials with a social media distractor, 24 trials with a social media distractor + notification symbol). In addition, to assess whether or not any effect of notification symbol was specific to the social media apps, notification symbols were also paired with four non-social media distractor apps selected from the set of 34 filler apps. In line with the social media distractors, these apps were present on 24 trials without a notification symbol and 24 trials in which it was present. Trial randomisation and counterbalancing was consistent with that described above for blocks 1 and 2. Upon completion of the attentional capture task, participants completed the questionnaire measures, with a typical testing session lasting approximately 45 minutes.

\section{Ethics}

This study was approved by the Ethics Committee of the University of Strathclyde School of Psychological Sciences and Health.

\section{RESULTS}

\section{Data analysis}

As response time (RT) is the primary measure for this task, and as no theoretically interesting effects arose from our error rate analysis, here we report RT effects. Only correct RTs were included in the final dataset. For condition 1, social media distractor app present vs. absent, an attentional bias is defined as an RT cost, which is calculated by subtracting RTs in which a social media distractor app was absent from RTs in which a social media distractor app was present. Positive RTs costs would therefore be indicative of an attentional bias. Similarly, for condition 2, distractor app vs. distractor app + notification, RT cost is calculated by subtracting RTs in which the notification was absent from RTs in which a notification was paired with a distractor app.

For the RT costs (i.e. the attentional bias) calculated in both condition 1 and condition 2, we assess whether individual differences in the size of the bias is predicted by social media use and whether there are any associations between it and scores on our established measures of social media engagement (SMES) and 'addictive' severity (BSNAS, SMAQ).

\section{Attentional bias condition 1 - Social media distractor apps present vs. absent}

First we assess whether there are any general social media attentional bias effects. That is, do social media users, in general, show an attentional bias for any social media distractor app regardless of whether they have an account with that platform or not. To that end, all participants mean RTs were entered into a one-way ANOVA with the withinsubjects factor of social media distractor app condition which has four levels (Facebook app present, Twitter app present, Instagram app present, Snapchat app present, and social media distractor app absent). The ANOVA revealed no main effect of distractor app condition, $F<1$, with no significant RT cost (i.e. attentional bias) for any of the social media platforms $(M=12 \mathrm{~ms}, S D=83 \mathrm{~ms}$ for Facebook; $M$ $=5 \mathrm{~ms}, S D=99 \mathrm{~ms}$ for Twitter; $M=-7 \mathrm{~ms}, S D=96 \mathrm{~ms}$ for Instagram; $M=-1 \mathrm{~ms}, S D=97 \mathrm{~ms}$ for Snapchat). That is, despite the fact that all participants in this sample were social media users, none of the social media apps generated an effect which would indicate the presence of an attentional bias towards those stimuli.

However, in order to rule out the possibility that the overall, all user, analysis did not mask any platform-specific effects, here we perform four separate one way ANOVAs with the within subjects factor of social media distractor app condition (present, absent) for each of the social media platforms individually. That is, each of the four ANOVAs include only users who have an account with that specific platform, and only RTs relating to the presence and absence of that distractor app (e.g. ANOVA 1, Facebook users only, Facebook distractor app present/absent RTs). As seen in Fig. 2, there was no evidence for a significant attentional bias effect when the platforms were considered individually, with non-significant main effects of distractor condition across the individual platform analysis, all $F$ 's $<1$. These findings suggest that, both at an overall group level, and for users of specific platforms, neither the presence of any social media distractor app, nor the presence of the app for which they have an account, captures attention to any greater extent than non-social media distractors.

\section{Individual differences condition 1}

Although there was no group or platform level attentional bias effects from the mere presence of the social media distractors, there was a large degree of variability in RT cost across participants. This could suggest that some participants, perhaps those who are the most frequent users, the 


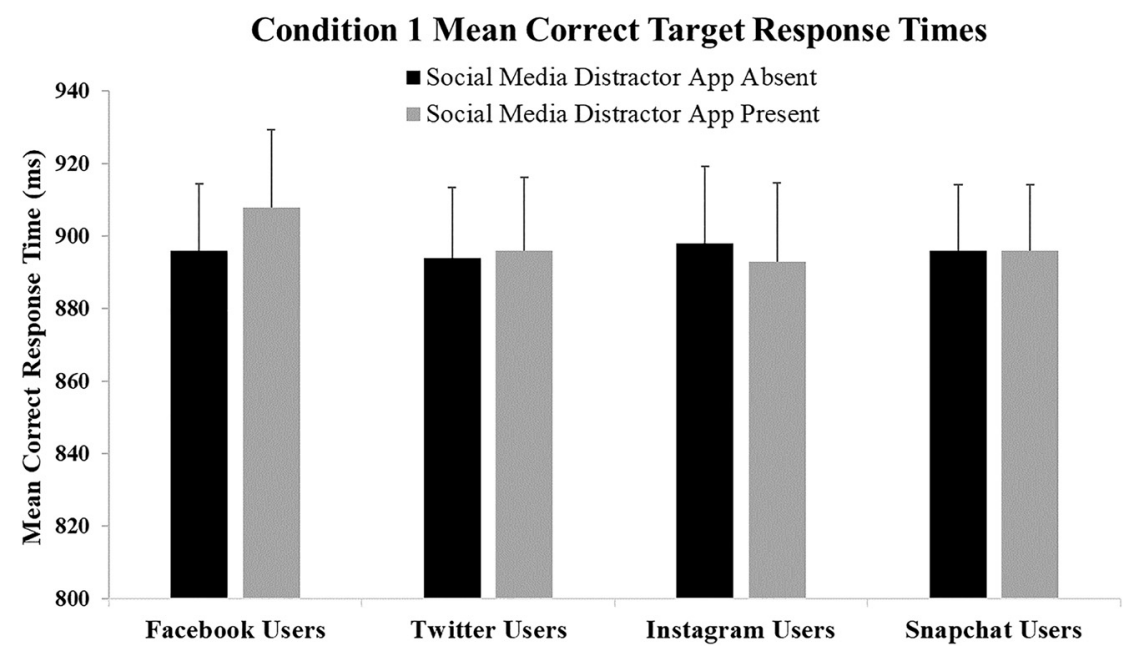

Fig. 2. Condition 1 mean correct target response times (RTs) in the absence of a social media distractor app (black) and the presence of a social media distractor app (grey; Facebook, Twitter, Instagram, Snapchat). Error bars denote standard error of the mean

most engaged, and who exhibit the greatest 'addictive' severity scores, could be demonstrating an attentional bias to social media stimuli, while more casual users might not. To assess this, separate multiple linear regressions were calculated for each of the social media platforms, using the predictor variables of amount of time spent on the app over the last week from the objective smartphone usage data, selfreported number of checks per day, number of posts per week, as well as number of friends/followers, summary data for these measures are provided in Table 1 . None of the overall models were found to be significant, all $F$ ' $s \leq 1.24$ all $P^{\prime} s \geq 0.304$, suggesting that these measures of social media use were not predictive of the extent to which an individual was likely to have their attention captured by a social media distractor app.

In addition to the self-report and objective engagement metrics reported above, we also recorded participant's scores on an established social media engagement questionnaire (SMES), and two 'addictive' severity scales (BSNAS, SMAQ), mean scores and variability are presented in Table 1 . A Pearson's correlation analysis showed that there were moderate-to-strong correlations across these three measures, suggesting that each of them is a robust questionnaire which tap individual differences in social media users $(r(98)=$ 0.782, $P<0.001$ for BSNAS/SMAQ; $r(97)=0.409, P<0.001$ for SMAQ/SMEC; $r(97)=0.507, P<0.001$ for SMAQ/ SEMC).

However, the important analysis assessed whether there were any associations between participant's scores on these measures, and the level of their social media distractor app RT cost. To that end, separate Person's correlations were calculated for each of the social media platforms and for each of the questionnaires, with a Bonferroni corrected alpha level of $0.004(0.05 / 12)$. As shown in Fig. 3, there were no significant correlations between RT cost and any of the questionnaire scores across all platforms.

That is, despite the large range of individual differences in the size of the attentional capture effect to social media distractor apps across participants, such differences were not predicted by, or associated with, self-reported, objective, or established measures of a participant's level of social media use, engagement, or 'addictive' severity.

\section{Attentional bias condition 2 - Distractor apps vs. distractor app + notification}

As with condition 1 we start by assessing general effects across all platform users. Participants mean RTs were entered into a $2 \times 5$ repeated measures ANOVA with the factors of notification (present, absent) and distractor type (non-social media app, Facebook app, Twitter app, Instagram app, Snapchat app). The ANOVA revealed a main effect of notification $F(1,98)=23.97, P<0.001, \eta_{p}{ }^{2}=$ 0.197 , with longer RTs for notification present trials $(M=$ $881 \mathrm{~ms})$ compared to notification absent trials $(M=845$ $\mathrm{ms}$ ), confirming that our notification manipulation was effective at capturing attention. There was also a main effect of distractor type, $F(4,392)=3.91, P=0.004, \eta_{p}{ }^{2}=0.038$, with numerically longer RTs for Facebook distractors compared each of the other distractor types, however, after the application of the Bonferroni correction for multiple comparisons (new alpha level $=0.005$ ), only the difference between Facebook app RTs $(M=886 \mathrm{~ms})$ and Twitter app RTs $(M=848 \mathrm{~ms})$ remained significant, $t(98)=3.44, P=$ 0.001 .

Importantly, the notification $\times$ distractor type interaction was not significant, $F(4,392)=1.35, P=0.252, \eta_{p}{ }^{2}=$ 0.014 , which shows that, at the overall user level, the RT notification cost was not larger in, or confined to, the social media distractor apps $(M=21 \mathrm{~ms}$ for non-social media distractors, $M=64 \mathrm{~ms}$ for Facebook, $M=23 \mathrm{~ms}$ for Twitter, $M=37 \mathrm{~ms}$ for Instagram, $M=32 \mathrm{~ms}$ for Snapchat). That is, despite small trends, particularly for Facebook, the magnitude of the RT cost found when a notification symbol was paired with a non-social media distractor app (e.g. Amazon, App Store, BBC iPlayer, 


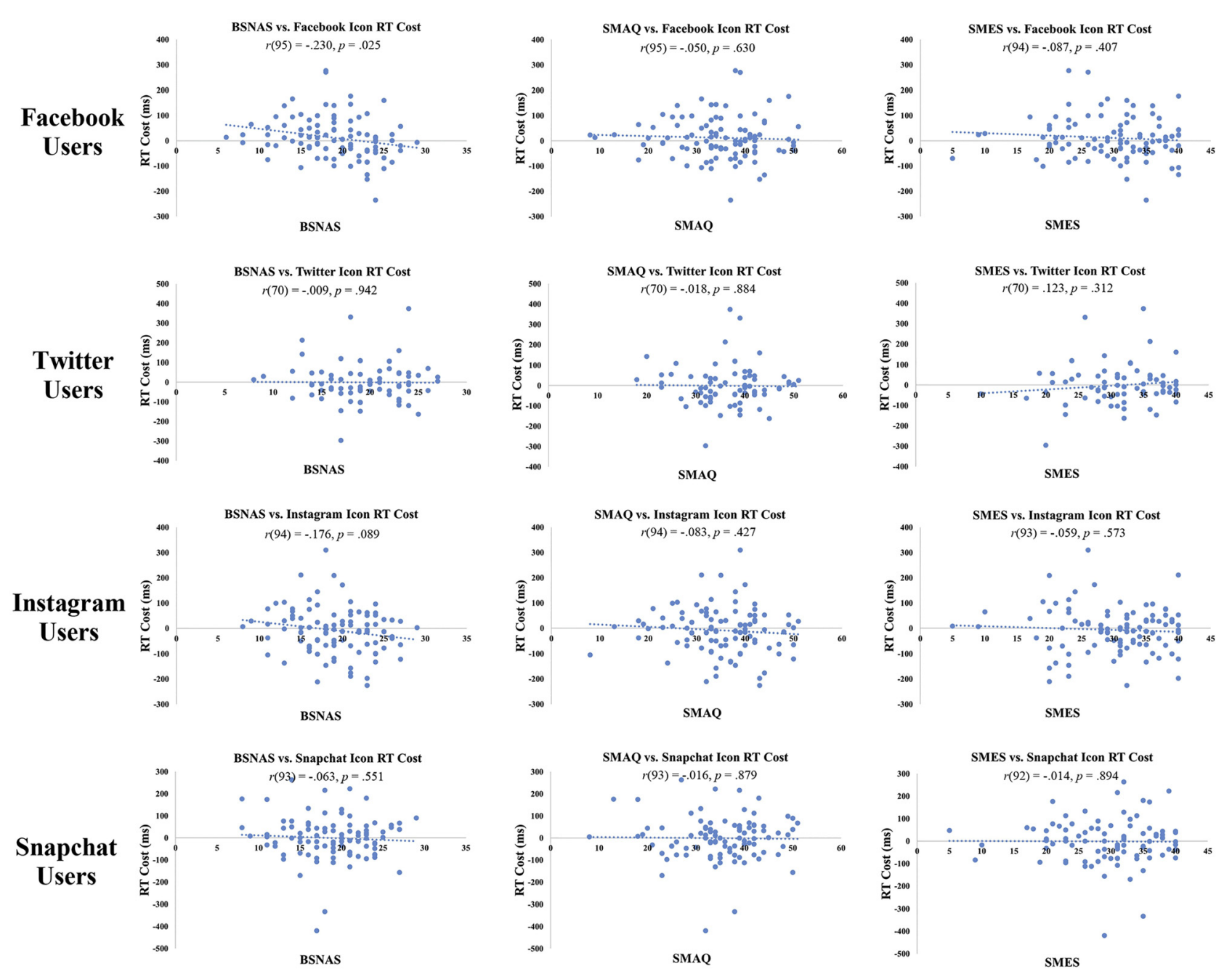

Fig. 3. Condition 1 scatterplots showing Pearson's correlation coefficients for the level of attentional capture by the presence of a social media distractor app (i.e. RT cost; social media distractor app present RT - social media distractor app absent) and scores on the established social media engagement (SMES) and 'addictive' severity scales (BSNAS, SMAQ).

Note: some of the $n$ values are lower than the total user sample size for each platform as a small number of participants did not complete all sections of all of the questionnaires)

Deliveroo) was not statistically different from the effect found when a notification symbol was paired with a social media app.

As with condition 1 we now focus on user and platform specific effects (i.e. Facebook users/Facebook App RTs/ Facbook App + Notification RTs). The platform specific ANOVAs revealed significant main effects of notification (present, absent) for each distractor type (non-social media distractor, specific platform distractor; all $P^{\prime} s \leq 0.007$ ), as seen in Fig. 4, and despite a trend for a numerically larger bias for Facebook distractor paired with a notification compared to the other distractor types, none of the notification condition $\times$ distractor type interactions were significant $(F,<1$ for all platforms apart from Facebook, $F(1,95)$ $\left.=3.66, P=0.059, \eta_{p}{ }^{2}=0.037\right)$. This finding shows that there would appear to be no difference in the perceptual processing 'weight' that is being attributed to notifications paired with social media stimuli, compared to those paired with non-social media stimuli.

\section{Individual differences condition 2}

In line with the individual differences analysis for condition 1 , individual differences in the time spent on the app over the last week, number of checks per day, number of posts per week, as well as number of friends/followers, were not predictive of individual differences in the magnitude of participants RT cost on trials in which a notification symbol was paired with a social media distractor app for which they had an account, all $F^{\prime} s \leq 1.00$, all $P^{\prime} s \geq 0.431$. In addition, Person's correlations, as shown in Fig. 5 , with a Bonferroni corrected alpha level of $0.004(0.05 / 12)$, produced no significant associations between RT cost in this condition and established measures of a participant's level of social media use, engagement, or 'addictive' severity.

\section{Additional analysis condition 2}

There were a number of participants who did not exhibit any attentional capture effect (i.e. RT costs $\leq 0 \mathrm{~ms}$ ) for the 


\section{Condition 2 Mean Correct Target Response Times}

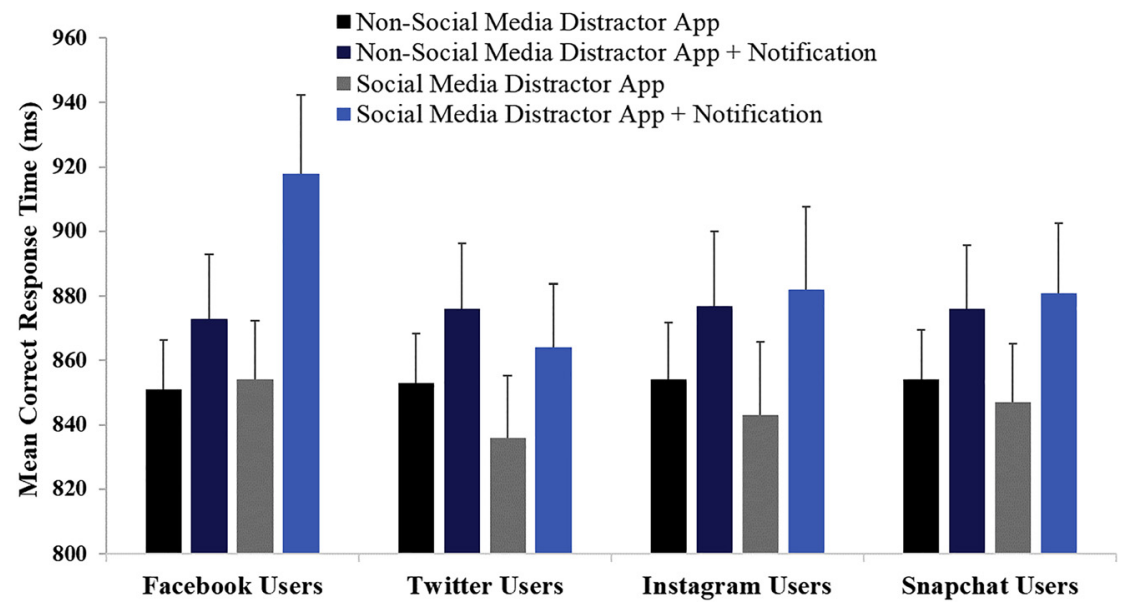

Fig. 4. Condition 2 mean correct target response times (RTs) in the non-social media distractor app condition (black), in the non-social media distractor app + notification condition (dark blue), in the social media distractor app condition (grey), and the social media distractor app + notification condition (light blue). Error bars denote standard error of the mean.

baseline condition in which a non-social media distractor app was paired with a notification despite the notification manipulation being successful at the group level. This could be due to the range of individual differences in the extent to which people are able to focus their attention on a primary task while ignoring task irrelevant information, in general, as shown most recently by Eayrs and Lavie (2019). It could therefore be the case that participants within the sample who did not exhibit an attentional capture effect by the red notification symbol (i.e. those who are better than average at focusing their attention), could be masking attentional bias effects among those in the sample who did exhibit the notification attentional capture effect.

To rule out this alternative explanation for the findings, we re-analysed the data using only participants who exhibited a minimum of a $10 \mathrm{~ms}$ RT cost for the presence of a notification for the non-social media distractors. Despite this, there was no significant change to the findings. Differences in RT cost for observing a notification paired with a social media distractor app compared to being paired with a non-social media distractor app remained negligible for Facebook $(M=72 \mathrm{~ms}$ for nonsocial media app, $M=78 \mathrm{~ms}$ for Facebook app; $t<1)$ and Instagram $(M=71 \mathrm{~ms}$ for non-social media app, $M=69$ $\mathrm{ms}$ for Instagram app; $t<1)$. For Twitter $(M=74 \mathrm{~ms}$ for non-social media app, $M=21 \mathrm{~ms}$ for Twitter app; $t(38)=$ $1.15, P=0.078)$ and Snapchat $(M=72 \mathrm{~ms}$ for non-social media app, $M=43 \mathrm{~ms}$ for Snapchat app; $t(51)=1.15, P=$ 0.255 ) the numerical differences did become larger in comparison to the original analysis, however, the change was in the opposite direction to that expected if the previous findings had been masking social media attentional bias effects (i.e. trends for smaller RT costs for a notification paired with a social media app compared to a notification paired with a non-social media app).

\section{DISCUSSION}

In this study we investigated whether an attentional bias to social media related stimuli might be present in a group of social media users. Using a task with high ecological validity, we tested whether social media users might show preferential attentional capture for social media apps and notifications. The sample was well powered and there was a large degree of variation in social media use, engagement, and 'addictive' severity as measured through self-reported platform use, objective smartphone data, and existing social media scales (BSNAS, SMAQ, SMES). Our findings show that the presence of social media distractor apps in mock smartphone displays had no effect on users' target response times, which in turn did not support the presence of an attentional bias to these stimuli.

This finding is in contrast to research showing attentional biases to addiction related stimuli in substance-related (see Field \& Cox, 2008 for a review) and non-substance related addictive behaviours. Ciccarelli et al. (2016), Jeromin et al. (2016), and Pekal et al. (2018) reported attentional biases to gambling related images, computer related words, and pornographic images in associated users. However, based on the findings from the present study, this does not appear to extend to the preferential processing of social media stimuli by social media users. If excessive social media use is to be viewed as an activity which might be placed within an addiction framework, we would have expected to find evidence of an attentional bias in this study. In contrast, here, within the context of social media use, we show no evidence for the hallmark change to attentional processes present in other addictive behaviours, which is thought to be driven by neurochemical changes to the areas of the brain that mediate goal directed behaviour (Franken, 2003; Robinson \& Berridge, 1993, 2008). 

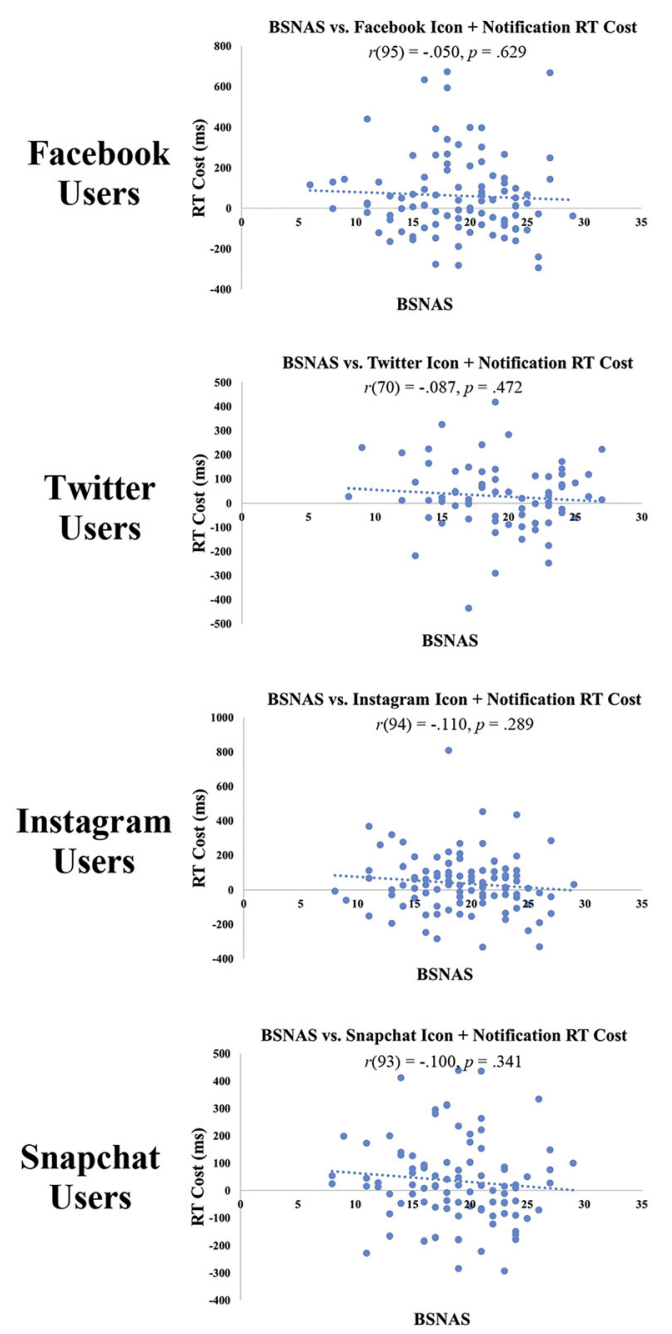
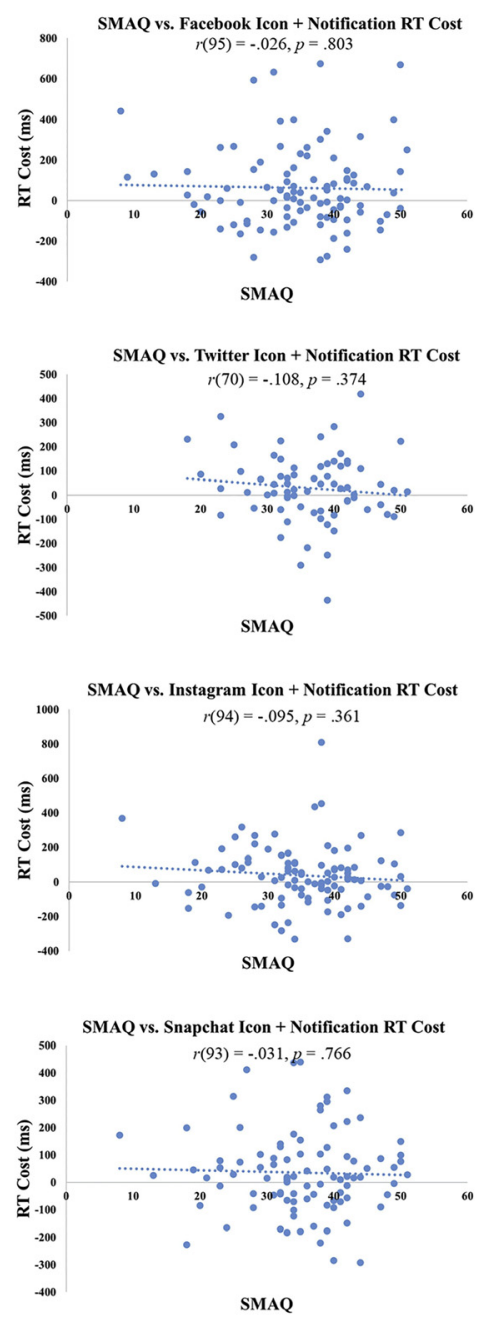
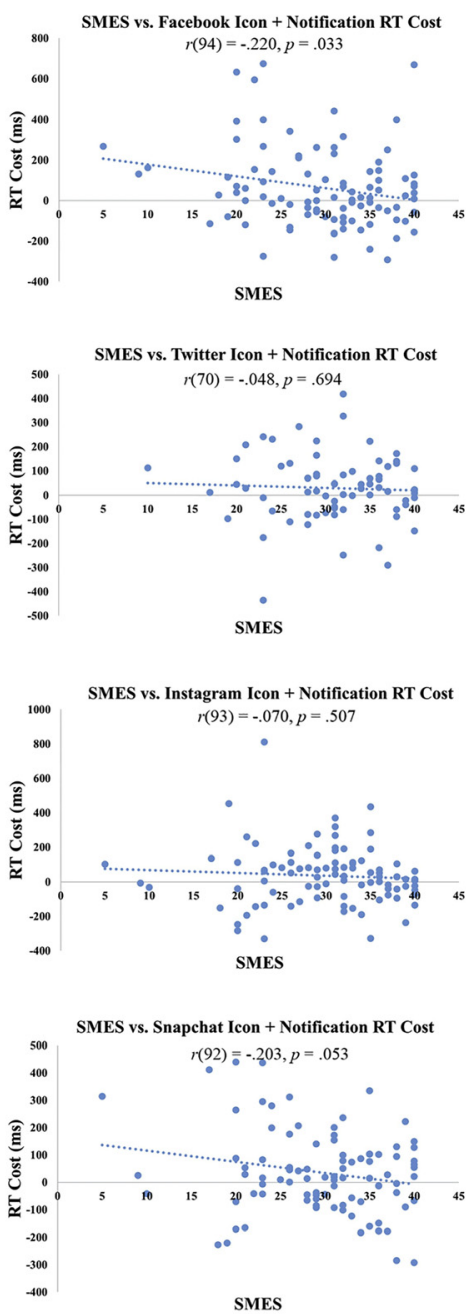

Fig. 5. Condition 2 scatterplots showing Pearson's correlation coefficients for the level of attentional capture by a social media distractor paired with a notification symbol (i.e. RT cost; social media distractor app + notification RT - social media distractor app RT) and scores on the established social media scales, the BSNAS (left), the SMAQ (middle), and the SMES (right), presented as a function of social media platform. Note: some of the $\mathrm{n}$ values are lower than the total user sample size for each platform as a small number of participants did not complete all sections of all of the questionnaires

While we demonstrated an attentional capture effect for the addition of a notification symbol to our distractor apps, the cost to target RTs was consistent regardless of whether the notification had been paired with a social media app or not. This is surprising given the importance of such notification symbols as indicators that 'Likes' or 'Comments' have been made to users' posts (Scissors et al., 2016). In contrast, here we show that social media notifications did not capture users' attention to any greater extent than, for example, a weather notification. While there was a trend for a larger RT cost for the Facebook notification in Facebook users, this could be due to non-social media specific effects such as the colour contrast (red notification, blue Facebook app; Theeuwes \& Godijn, 2002), or a general familiarity effect to this logo, which is the oldest of the platform logos, and could therefore have been generated by ubiquitous exposure to it (see Qin, Koutstaal, \& Engel, 2014).

In line with the lack of an attentional bias effect, our individual differences analyses also did not yield any associations between social media use, engagement, or 'addictive' severity. Time spent using the app, number of checks per day, number of posts per week, and number of friends/followers were not predictive of the magnitude of user's level of attentional capture by social media distractors, nor were there any associations between the level of attentional capture and scores on the established social media engagement and 'addictive' severity scales (SMES, BSNAS, SMAQ). Again, this is in contrast to previous work on nonsubstance related addictions as shown by Ciccarelli et al. (2016; Gambling), Jeromin et al. (2016; Gamers), and Pekal et al. (2018; Internet Pornography Use), which reported positive associations between levels of attention bias and use/ severity measures. Moreover, while Nikolaidou et al. (2019) did report an association between the level of urge to be online and attentional dwell time to social media related stimuli, this effect was based on a small sample size $(N=16)$.

One interesting reason why we may not have found an attentional bias and associated relationship with use, 
engagement, and severity measures, relates to a recent review of this literature by Field et al. (2016). Field et al. (2016) argue that research on substance-users does not consistently yield evidence for a robust attentional bias effect. They argue that, rather than such a bias being a core aspect of addictive behaviour, that it would be better characterised as momentary changes in the evaluation of the addiction related stimuli during periods of subjective craving ('an appetitive motivational process'). When applying this to social media use, the period of subjective craving is actually most likely to be the period of time after a user has posted an item of content. It is likely to be during this period that the incentive salience of the social media app and notification symbol are likely to be at their greatest, as participants seek their behavioural reward (i.e. likes and comments to their post). It might therefore be the case that a social media attentional bias is a transient effect which, if the effect exists, is mostly likely to be detected after a user has posted content for which the reward is peer feedback.

Therefore, future research should investigate whether a social media attentional bias exists after the user/participant has been asked to post content for which they place value on the number of likes/comments returned. In addition, while the present study used a variety of social media use, engagement, and 'addictive' severity questionnaires, a new measure should be developed which explicitly refers to the level of 'craving' for social media access, and for social media validation in the form of a 'craving' for likes and comments (see Savci \& Griffiths, 2019). This would bring the individual difference measures used in relation to social media use in line with other addiction related questionnaires. Similarly, while our sample did capture a large range of individual differences in social media use, engagement and 'addictive' severity, future research should seek to work on setting out common criteria which would allow for the categorisation of excessive social media use, in the sense ascribed to substance-related and other non-substance related addictive behaviours. This would enhance the ability of future studies to run individual differences analyses not just for potential attentional bias effects, but also for the further assessment of the positive and negative effects of such use, as outlined in the introduction (see also Wegmann, Müller, Turel, \& Brand, 2020).

In summary, social media use has become a ubiquitous part of society. Research has largely focused on the negative outcomes which may be associated with excessive use, and there is a growing debate which relates to whether this should be categorised as a clinically relevant addictive behaviour. An attentional bias to addiction related stimuli is considered to be a hallmark of the changes to brain neurochemistry and attentional deployment which arise from addictive behaviours. While there is evidence for such biases in both substance and non-substance related addictions, we found no such effect in relation to social media stimuli in a varied group of social media users. Future research should focus on whether such a bias may exist in alternative contexts, as outlined above, and to generate a novel and comprehensive measure for the assessment of individual differences in social media use, engagement, 'addictive' severity, and craving.

Funding sources: No financial support was received for this study.

Authors' contribution: Study, concept and design (DJR, KT, $\mathrm{SCH}, \mathrm{SHB})$, data collection $(\mathrm{KT})$, statistical analysis (KT, $\mathrm{DJR})$, interpretation of data (KT, DJR, SCH, SHB), contributed to the manuscript (DJR, KT, SCH, SHB).

Conflicts of Interest: The authors declare no conflict of interest.

Acknowledgments: Our thanks to Hannah Geddis who assisted with data collection.

\section{REFERENCES}

Allen, K. A., Ryan, T., Gray, D. L., McInerney, D. M., \& Waters, L. (2014). Social media use and social connectedness in adolescents: The positives and the potential pitfalls. The Educational and Developmental Psychologist, 31(1), 18-31. https://doi.org/ 10.1017/edp.2014.2.

American Psychiatric Association. (2013). Diagnostic and statistical manual of mental disorders (5th ed.). https://doi.org/10.1176/ appi.books.9780890425596.

Anderson, B. A. (2013). A value-driven mechanism of attentional selection. Journal of Vision, 13(3), 1-7. https://doi.org/10. 1167/13.3.7.

Andreassen, C. S., Billieux, J., Griffiths, M. D., Kuss, D. J., Demetrovics, Z., Mazzoni, E., et al. (2016). The relationship between addictive use of social media and video games and symptoms of psychiatric disorders: A large-scale cross-sectional study. Psychology of Addictive Behaviors, 30(2), 252-262. http://dx.doi. org/10.1037/adb0000160.

Andreassen, C. S., Pallesen, S., \& Griffiths, M. D. (2017). The relationship between addictive use of social media, narcissism, and selfesteem: Findings from a large national survey. Addictive Behaviours, 64, 287-293. https://doi.org/10.1016/j.addbeh.2016.03.006.

Andreassen, C. S., Torsheim, T., Brunborg, G. S., \& Pallesen, S. (2012). Development of a Facebook addiction scale. Psychological Reports, 110, 501-517. https://doi.org/10.2466/02.09.18. PR0.110.2.501-517.

Bányai, F., Zsila Á., Király, O., Maraz, A., Elekes, Z., Griffiths, M. D., et al. (2017). Problematic social media use: Results from a largescale nationally representative adolescent sample. PloS One, 12(1), e0169839. https://doi.org/10.1371/journal.pone.0169839.

Boyer, M., \& Dickerson, M. (2003). Attentional bias and addictive behaviour: Automaticity in a gambling-specific modified Stroop task. Addiction, 98(1), 61-70. https://doi.org/10.1046/j.13600443.2003.00219.x.

Brand, M., Wegmann, E., Stark, R., Müller, A., Wölfling, K., Robbins, T. W., et al. (2019). The Interaction of Person-Affect- 
Cognition-Execution (I-PACE) model for addictive behaviors: Update, generalization to addictive behaviors beyond internetuse disorders, and specification of the process character of addictive behaviors. Neuroscience and Biobehavioral Reviews, 104, 1-10. https://doi.org/10.1016/j.neubiorev.2019.06.032.

Brown, J., Beard, E., Kotz, D., Michie, S., \& West, R. (2014). Realworld effectiveness of e-cigarettes when used to aid smoking cessation: A cross-sectional population study. Addiction, 109(9), 1531-1540. https://doi.org/10.1111/add.12623.

Carter, B. L., \& Tiffany, S. T. (1999). Meta-analysis of cue-reactivity in addiction research. Addiction, 94(3), 327-340. https://doi. org/10.1046/j.1360-0443.1999.9433273.x.

Ciccarelli, M., Nigro, G., Griffiths, M. D., Cosenza, M., \& D’Olimpio, F. (2016). Attentional biases in problem and non-problem gamblers. Journal of Affective Disorders, 198, 135-141.

Coyne, S. M., Rogers, A. A., Zurcher, J. D., Stockdale, L., \& Booth, M. (2020). Does time spent using social media impact mental health?: An eight year longitudinal study. Computers in Human Behavior, 104, 106160. https://doi.org/10.1016/j.chb.2019.106160.

Drummond, D. C. (2000). What does cue-reactivity have to offer clinical research? Addiction, 95(8s2), 129-144.

Eayrs, J. O., \& Lavie, N. (2019). Individual differences in parietal and frontal cortex structure predict dissociable capacities for perception and cognitive control. Neuroimage, 202, 116148. https://doi.org/10.1016/j.neuroimage.2019.116148.

Ellis, D. A., Davidson, B. I., Shaw, H., \& Geyer, K. (2019). Do smartphone usage scales predict behavior? International Journal of Human-Computer Studies, 130, 86-92. https://doi.org/10. 1016/j.ijhcs.2019.05.004.

Enoch, M. A., \& Goldman, D. (2002). Problem drinking and alcoholism: Diagnosis and treatment. American Family Physician, 65(3), 441-449. https://www.aafp.org/afp/2002/0201/ p441.html.

Field, M., \& Cox, W. M. (2008). Attentional bias in addictive behaviors: A review of its development, causes, and consequences. Drug and Alcohol Dependence, 97(1-2), 1-20. https://doi.org/ 10.1016/j.drugalcdep.2008.03.030.

Field, M., Werthmann, J., Franken, I., Hofmann, W., Hogarth, L., \& Roefs, A. (2016). The role of attentional bias in obesity and addiction. Health Psychology, 35(8), 767-780. https://doi.org/ 10.1037/hea0000405.

Franken, I. H. (2003). Drug craving and addiction: Integrating psychological and neuropsychopharmacological approaches. Progress in Neuro-Psychopharmacology and Biological Psychiatry, 27(4), 563-579.

Gainsbury, S. M. (2015). Online gambling addiction: The relationship between internet gambling and disordered gambling. Current Addiction Reports, 2(2), 185-193. https://doi.org/10. 1007/s40429-015-0057-8.

Griffiths, M. (2000). Does internet and computer" addiction" exist? Some case study evidence. CyberPsychology and Behavior, 3(2), 211-218. https://doi.org/10.1089/109493100316067.

Griffiths, M. (2005). A “components" model of addiction within a biopsychosocial framework. Journal of Substance Use, 10(4), 191-197.

Griffiths, M. D. (2018). Common myths in the behavioral addiction field. Journal of Concurrent Disorders, 1(1), 128-141. http:// irep.ntu.ac.uk/id/eprint/34099.
Griffiths, M. D., \& Kuss, D. (2017). Adolescent social media addiction (revisited). Education and Health, 35(3), 49-52. http://sheu.org.uk/sheux/EH/eh353mg.pdf.

Haber, S. N. (2016). Corticostriatal circuitry. Dialogues in Clinical Neuroscience, 18(1), 7. https://doi.org/10.31887/DCNS.2016.18. $1 /$ shaber.

Hawi, N. S., \& Samaha, M. (2017). The relations among social media addiction, self-esteem, and life satisfaction in university students. Social Science Computer Review, 35(5), 576-586. https://doi.org/10.1177/0894439316660340.

Heffer, T., Good, M., Daly, O., MacDonell, E., \& Willoughby, T. (2019). The longitudinal association between social-media use and depressive symptoms among adolescents and young adults: An empirical reply to Twenge et al. (2018). Clinical Psychological Science, 7(3), 462-470. https://doi.org/10.1177/ 2167702618812727.

van Holst, R. J., Lemmens, J. S., Valkenburg, P. M., Peter, J., Veltman, D. J., \& Goudriaan, A. E. (2012). Attentional bias and disinhibition toward gaming cues are related to problem gaming in male adolescents. Journal of Adolescent Health, 50(6), 541-546. https://doi.org/10.1016/j.jadohealth.2011.07.006.

Houghton, S., Lawrence, D., Hunter, S. C., Rosenberg, M., Zadow, C., Wood, L., et al. (2018). Reciprocal relationships between trajectories of depressive symptoms and screen media use during adolescence. Journal of Youth and Adolescence, 47(11), 2453-2467. https://doi.org/10.1007/s10964-018-0901-y.

Jeromin, F., Nyenhuis, N., \& Barke, A. (2016). Attentional bias in excessive Internet gamers: Experimental investigations using an addiction Stroop and a visual probe. Journal of Behavioral Addictions, 5(1), 32-40. https://doi.org/10.1556/2006.5.2016.012.

Jones, B. T., Bruce, G., Livingstone, S., \& Reed, E. (2006). Alcoholrelated attentional bias in problem drinkers with the flicker change blindness paradigm. Psychology of Addictive Behaviors, 20(2), 171-177. https://doi.org/10.1037/0893-164X.20.2.171.

Kaye, L. K., Orben, A., Ellis, D. A., Hunter, S. C., \& Houghton, S. (2020). The conceptual and methodological mayhem of "screen time". International Journal of Environmental Research and Public Health, 17(10), 3661. https://doi.org/10.3390/ijerph17103661.

Keles, B., McCrae, N., \& Grealish, A. (2020). A systematic review: The influence of social media on depression, anxiety and psychological distress in adolescents. International Journal of Adolescence and Youth, 25(1), 79-93. https://doi.org/10.1080/ 02673843.2019.1590851.

Kuss, D. J., \& Griffiths, M. D. (2012). Internet gaming addiction: A systematic review of empirical research. International Journal of Mental Health and Addiction, 10(2), 278-296. https://doi.org/ 10.1007/s11469-011-9318-5.

Kuss, D. J., \& Griffiths, M. D. (2017). Social networking sites and addiction: Ten lessons learned. International Journal of Environmental Research and Public Health, 14(3), 311. https://doi. org/10.3390/ijerph14030311.

Kuss, D. J., \& Lopez-Fernandez, O. (2016). Internet addiction and problematic internet use: A systematic review of clinical research. World Journal of Psychiatry, 6(1), 143-176. https:// doi.org/10.5498/wjp.v6.i1.143.

Kırcaburun, K., Kokkinos, C. M., Demetrovics, Z., Király, O., Griffiths, M. D., \& Çolak, T. S. (2018). Problematic online behaviors among adolescents and emerging adults: Associations 
between cyberbullying perpetration, problematic social media use, and psychosocial factors. International Journal of Mental Health and Addiction, 17(4), 891-908. https://doi.org/10.1007/ s11469-018-9894-8.

LaRose, R., Kim, J., \& Peng, W. (2010). Social networking: Addictive, compulsive, problematic, or just another media habit?. In Z. Papacharissi (Ed.) A networked self (pp. 67-89). New York, NY: Routledge.

Leist, A. K. (2013). Social media use of older adults: A mini-review. Gerontology, 59(4), 378-384. https://doi.org/10.1159/000346818.

Lin, L. Y., Sidani, J. E., Shensa, A., Radovic, A., Miller, E., Colditz, J. B., et al. (2016). Association between social media use and depression among US young adults. Depression and Anxiety, 33(4), 323-331. https://doi.org/10.1002/da.22466.

McDaniel, B. T., Coyne, S. M., \& Holmes, E. K. (2012). New mothers and media use: Associations between blogging, social networking, and maternal well-being. Maternal and Child Health Journal, 16(7), 1509-1517. https://doi.org/10.1007/ s10995-011-0918-2.

Nikolaidou, M., Fraser, D. S., \& Hinvest, N. (2019). Attentional bias in Internet users with problematic use of social networking sites. Journal of Behavioral Addictions, 8(4), 733-742. https:// doi.org/10.1556/2006.8.2019.60.

Papachristou, H., Nederkoorn, C., Giesen, J. C., \& Jansen, A. (2014). Cue reactivity during treatment, and not impulsivity, predicts an initial lapse after treatment in alcohol use disorders. Addictive Behaviors, 39(3), 737-739. https://doi.org/10.1016/j. addbeh.2013.11.027.

Pekal, J., Laier, C., Snagowski, J., Stark, R., \& Brand, M. (2018). Tendencies toward Internet-pornography-use disorder: Differences in men and women regarding attentional biases to pornographic stimuli. Journal of Behavioral Addictions, 7(3), 574-583. https://doi.org/10.1556/2006.7.2018.70.

Przybylski, A. K., Murayama, K., DeHaan, C. R., \& Gladwell, V. (2013). Motivational, emotional, and behavioral correlates of fear of missing out. Computers in Human Behavior, 29(4), 1841-1848. https://doi.org/10.1016/j.chb.2013.02.014.

Qin, X. A., Koutstaal, W., \& Engel, S. A. (2014). The hard-won benefits of familiarity in visual search: Naturally familiar brand logos are found faster. Attention, Perception, \& Psychophysics, 76(4), 914-930. https://doi.org/10.3758/s13414-014-0623-5.

Robbins, S. J., \& Ehrman, R. N. (2004). The role of attentional bias in substance abuse. Behavioral and Cognitive Neuroscience Reviews, 3(4), 243-260. https://doi.org/10.1177/1534582305275423.

Robinson, T. E., \& Berridge, K. C. (1993). The neural basis of drug craving: An incentive-sensitization theory of addiction. Brain Research Reviews, 18(3), 247-291. https://doi.org/10.1016/01650173(93)90013-P.

Robinson, T. E., \& Berridge, K. C. (2008). The incentive sensitization theory of addiction: Some current issues. Philosophical Transactions of the Royal Society B: Biological Sciences, 363(1507), 3137-3146. https://doi.org/10.1098/rstb.2008.0093.
Ryan, T., Chester, A., Reece, J., \& Xenos, S. (2014). The uses and abuses of Facebook: A review of Facebook addiction. Journal of Behavioral Addictions, 3(3), 133-148. https://doi.org/10.1556/ jba.3.2014.016.

Savci, M., \& Griffiths, M. D. (2019). The development of the Turkish social media craving scale (SMCS): A validation study. International Journal of Mental Health and Addiction, 1-15. https://doi.org/10.1007/s11469-019-00062-9.

Scissors, L., Burke, M., \& Wengrovitz, S. (2016, February). What's in a like? Attitudes and behaviors around receiving likes on Facebook. In Proceedings of the 19th ACM conference on computer-supported cooperative work \& social computing (pp. 1501-1510). https://doi.org/10.1145/2818048.2820066.

Sharma, D., Albery, I. P., \& Cook, C. (2001). Selective attentional bias to alcohol related stimuli in problem drinkers and nonproblem drinkers. Addiction, 96(2), 285-295. https://doi.org/10. 1046/j.1360-0443.2001.96228512.x.

Shensa, A., Escobar-Viera, C. G., Sidani, J. E., Bowman, N. D., Marshal, M. P., \& Primack, B. A. (2017). Problematic social media use and depressive symptoms among US young adults: A nationally-representative study. Social Science \& Medicine, 182, 150-157. https://doi.org/10.1016/j.socscimed.2017.03.061.

Sofuoglu, M., \& Kosten, T. R. (2006). Emerging pharmacological strategies in the fight against cocaine addiction. Expert Opinion on Emerging Drugs, 11(1), 91-98. https://doi.org/10.1517/ 14728214.11.1.91.

Statista (2020). Social media usage worldwide. Retrieved from https://www.statista.com/study/12393/social-networks-statistadossier/on $1^{\text {st }}$ March 2020.

Theeuwes, J., \& Godijn, R. (2002). Irrelevant singletons capture attention: Evidence from inhibition of return. Perception \& Psychophysics, 64(5), 764-770. https://doi.org/10.3758/BF03194743.

Waters, A. J., Shiffman, S., Sayette, M. A., Paty, J. A., Gwaltney, C. J., \& Balabanis, M. H. (2003). Attentional bias predicts outcome in smoking cessation. Health Psychology, 22(4), 378-387. https://doi.org/10.1037/0278-6133.22.4.378.

Wegmann, E., Müller, S. M., Turel, O., \& Brand, M. (2020). Interactions of impulsivity, general executive functions, and specific inhibitory control explain symptoms of social-networksuse disorder: An experimental study. Scientific Reports, 10(1), 1-12. https://doi.org/10.1038/s41598-020-60819-4.

Whiting, A., \& Williams, D. (2013). Why people use social media: A uses and gratifications approach. Qualitative Market Research: An International Journal, 16(4), 362-369. https://doi. org/10.1108/QMR-06-2013-0041.

Woods, H. C., \& Scott, H. (2016). \# Sleepyteens: Social media use in adolescence is associated with poor sleep quality, anxiety, depression and low self-esteem. Journal of Adolescence, 51, 41-49.

World Health Organization. (2018). International classification of diseases for mortality and morbidity statistics (11th Revision). https://icd.who.int/browse11/1-m/en.

\footnotetext{
Open Access. This is an open-access article distributed under the terms of the Creative Commons Attribution-NonCommercial 4.0 International License (https:// creativecommons.org/licenses/by-nc/4.0/), which permits unrestricted use, distribution, and reproduction in any medium for non-commercial purposes, provided the original author and source are credited, a link to the CC License is provided, and changes - if any - are indicated.
} 